\title{
EVALUATION OF DIGITAL FILTERS FOR SIMILARITY ANALYSIS BETWEEN TOMOSYNTHESIS AND 2D MAMMOGRAPHIC IMAGES
}

\author{
P. C. Carneiro*, G. D. Gallucci*, V. S. Rocha*, C. L. Debs**, N. Barros***, A. O. Andrade*, A. C. \\ Patrocinio*
*Faculty of Electrical Engineering, Federal University of Uberlândia, Uberlândia, Brazil. **Faculty of Medicine, Federal University of Uberlândia, Uberlândia, Brazil. ***Faculty of Medicine, University of São Paulo, São Paulo, Brazil.

e-mail: pedrocarneiro@ufu.br

\begin{abstract}
Breast cancer is the second leading cause of cancer death in women. Tomosynthesis is a new technique of additional exam that was created in order to improve the early detection of such disease. This technique shows a reconstructed image from different projections of the breast, reducing, this way, the effect of quantum noise due to the small thickness of the object of study. This paper aims at comparing the $3 D$ reconstructed image with some filtered $2 D$ polymethylmethacrylate (PMMA) images using structural similarity index (SSIM). We tested Wiener, Non-local Means, and Adaptive Median digital filters, which were applied at a region of interest of a $2 D$ conventional mammography acquired in combo mode. From this work, we could quantify the level of similarity from different digital filters. Wiener and Adaptive Median filters increased the similarity between the $2 D$ and the 3D tomosynthesis image, in terms of luminance, contrast and structure. However, the image with Nonlocal Means decreased significantly the similarity between the pair of compared images, proving the ineffective of this filter in reducing quantum noise.
\end{abstract}

Keywords: 2D mammography, digital filter, similarity, SSIM, tomosynthesis.

\section{Introduction}

For the past few years, while aiming for an earlier detection of breast cancer, a new and promising technique of digital image acquisition has been created: tomosynthesis [1]. On this new technique, commonly referred to as 3D mammography, the X-ray tube is rotated in a single plan around the compressed breast, generating a series of projections, one for each angulation of the X-ray tube. Thus, many slices are produced, that is, images in thin cuts of the breast from the series of projections that are generated [2-3].

When using this system, tumors are identified more easily, specially in dense breasts, due to a higher level of detail provided by this type of image and a higher contrast variation compared to the conventional mammography 2D images [2, 4-6]. A way to enhance certain 2D mammograms characteristics is by using digital filters that remove undesirable signal, mainly the quantum noise, improving the image assessment [7-9].

The quality of the images can be assessed quantitatively with the help of several similarity indexes described in scientific literature [10-14]. The structural similarity (SSIM) algorithm is regarded as well consolidated. It is broadly accepted due to its great evaluation precision and simple mathematical formulation [14].

The aim of this paper is to quantitatively compare the $3 \mathrm{D}$ reconstructed image (used as reference) with filtered 2D polymethylmethacrylate (PMMA) images, evaluating the influence of the filters used, through the structural similarity index (SSIM).

\section{Materials e methods}

For the image acquisition, we used a Full Field Digital Mammography from Hologic Inc, Selenia Dimensions model that has the tomosynthesis technology included for image acquisition. The images were acquired at Radiology Institute of the University of São Paulo using polymethylmethacrylate (PMMA) plates totaling $50 \mathrm{~mm}$ of thickness.

This equipment can produce the combined $3 \mathrm{D}$ and 2D images, known as combo mode, an imaging mode whereby both a $3 \mathrm{D}$ and $2 \mathrm{D}$ digital mammography image set are acquired in one breast compression.

For the tests, we selected two images in the combo mode of the same exam $(30 \mathrm{kVp})$ : conventional $2 \mathrm{D}$ image and the $3 \mathrm{D}$ reconstruction generated from the 15 projections produced in the PMMA plates. The conventional $2 \mathrm{D}$ image is nothing more than a "projection" acquired with the X-ray tube positioned in a zero degree angle.

After the image acquisition, the 2D image was the only one post-processed with digital filters, since it is known that this type of image is affected by quantum noise. Digital filtering consists in applying operators/masks which aims at softening, correcting or enhancing an image characteristic on a certain specific application, trying to reduce undesirable artifacts of the image. 
In order to evaluate the digital filtering influence, three filters were applied to the 2D image: Wiener filter, Non-local Means filter and an Adaptive Median filter. Thus, four comparisons were made, such as: 3D tomosynthesis and conventional 2D (without postprocessing), and 3D tomosynthesis with each one of the 2D filtered images (Wiener, Non-local Means, Adaptive Median).

The Wiener filter, also called the root mean square error filter, is an optimal linear filter and adaptive lowpass. The coefficients of this type of filter are calculated to minimize the square of the average distance between the filter result and the desired sign [15]. On this paper, we applied the Wiener filter on the conventional $2 \mathrm{D}$ image with a fixed window of $3 \times 3$ pixels.

The Non-local Means filter was the second one applied. Instead of behaving like most of the conventional algorithms, Non-local Means carries out a non-local filtering in which, for every pixel, all the other image pixels are taken into account to perform the filtering [16].

The other filter tested was the Adaptive Median with a fixed window of $3 \times 3$ pixels, based on the same concepts of the median filter. Nevertheless, the difference is on the sliding mask, which is applied in an adaptive way for every point of the image. The adaptation process is carried out for each one of the four axis used on the mask and it consists of the determination of the variation on the value of grey level inside the pre-established window [17].

Due to the different image dimensions, a region of interest (ROI) was defined for each of the five images tested this study. In order to do so, the central point of each image was calculated and a square region of $100 \times 100$ pixels was defined from this central coordinate, shown in Figure 1.
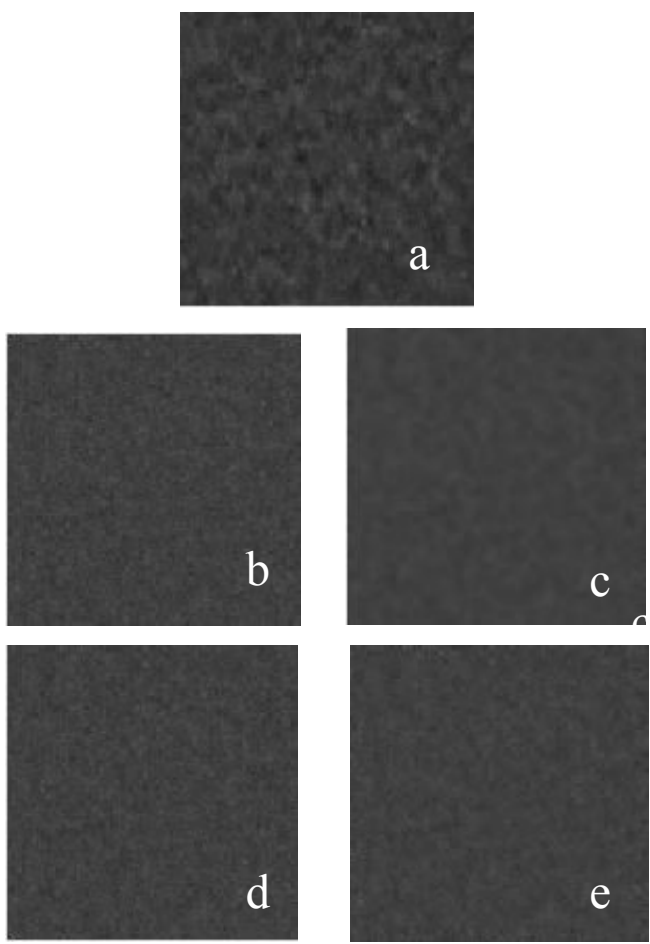

Figure 1: Regions of interest: (a) 3D image; (b) 2D image (conventional); (c) 2D Wiener; (d) 2D Non-local Means; (e) 2D Adaptive Median.

After defining the regions of interest (ROIs), the next step is to implement the structural similarity (SSIM) index. Such SSIM index was chosen because it has been broadly used; it is precise on its evaluations, as well as having simple mathematical formulation and short processing time [12]. The filters and similarity algorithm has been developed on the MATrix LABoratory (MATLAB $\AA$ ) software.

The SSIM index is based on the comparison of pixel intensity local patterns, which are standardized to luminance, contrast and structure (covariance) [14]. Such similarity measure is calculated on several image windows, where the window size and function can be defined by the user. The similarity index between two windows of $\mathrm{x}$ and $\mathrm{y}$ coordinates and of the same size is presented as follows on Equation 1.

$$
\operatorname{SSIM}(x, y)=\frac{\left(2 \mu_{x} \mu_{y}+C_{1}\right)\left(2 \sigma_{x y}+C_{2}\right)}{\left(\mu_{x}^{2}+\mu_{y}^{2}+C_{1}\right)\left(\sigma_{x}^{2}+\sigma_{y}^{2}+C_{2}\right)}
$$

On Equation 1, $\mu \mathrm{x}$ and $\mu \mathrm{y}$ correspond to the average of coordinates $\mathrm{x}$ and $\mathrm{y} ; \sigma \mathrm{x}$ and $\sigma \mathrm{y}$, to the $\mathrm{x}$ and $\mathrm{y}$ variance; $\sigma \mathrm{xy}$, to the $\mathrm{x}$ and $\mathrm{y}$ covariance; and, finally, $\mathrm{C} 1$ and $\mathrm{C} 2$, two constants which stabilize the division when $\mu \mathrm{x}$ and $\mu \mathrm{y}$ are close to zero. $\mathrm{C} 1$ and $\mathrm{C} 2$ depend on the square of the multiplication of the dynamic range of $\mathrm{L}$ pixels by a constant vector defined by default as 0.05 .

The L variable, then, equals to $\left(2^{\text {Number of bits }}-1\right)$, that is, if the image is 8 bits, L equals to 255 . In our case, the L value was kept 1023, as the images were 10 bits.

In practice, only an image global quality measure is needed. This way, it is used the Mean Structural Similarity (MSSIM) of the resulting matrix; it is composed by the SSIM index calculated between the $\mathrm{X}$ and $\mathrm{Y}$ images compared, from each window scanning, presented by Equation 2. The comparison can be made between any two images, allowing several applications [18].

$$
\operatorname{MSSIM}(X, Y)=\frac{1}{M} \sum_{j=1}^{M} \operatorname{SSIM}\left(x_{j} y_{j}\right)
$$

On Equation 2, the $M$ variable indicates the number of the image local windows and $x j$ and $y j$ indicate the image content on the $\mathrm{j}$ position of the local window. The implemented MSSIM value varies between 0 and 1, where the higher the MSSIM value is, the more similar one image is to the other. The value 1 is reached when the compared image pair is identical.

The Mean Structural Similarity (MSSIM) was calculated between the pairs of ROIs (Figure 1) 
analyzed using the Gaussian function (low-pass filter, $\sigma$ $=1.5$ ).

\section{Results}

Table 1 shows the results obtained to the similarity index MSSIM from the tests with the PMMA images. We used a Gaussian windowing function $(\sigma=1.5)$ with a window of size $3 \times 3$ pixels.

Table 1: Values obtained for the MSSIM calculated for each pair of ROIs compared.

\begin{tabular}{cc}
\hline MSSIM & $\begin{array}{c}\text { Gaussian } \\
\boldsymbol{\sigma}=\mathbf{1 . 5}\end{array}$ \\
\hline 3D vs. 2D & 0.7992 \\
3D vs. 2D Wiener & 0.8678 \\
3D vs. 2D Non-local Means & 0.1583 \\
3D vs. 2D Adaptive Median & 0.8326 \\
\hline
\end{tabular}

The similarity index between the conventional 2D image and the 3D tomosynthesis image was 0.7992 . With this value, we can analyze the influence of the filters applied on the 2D conventional image.

After using the Wiener and the Adaptive Median filters, the index reached the highest similarity values, 0.8678 and 0.8326 , respectively. The lowest similarity value was 0.1583 , when the Non-local Means filter was applied on 2D image.

Besides the quantitave analysis of the MSSIM values of each comparison, a similarity map of each analyzed image pair was also generated, allowing a visual analysis. Figure 2 shows the similarity maps obtained.

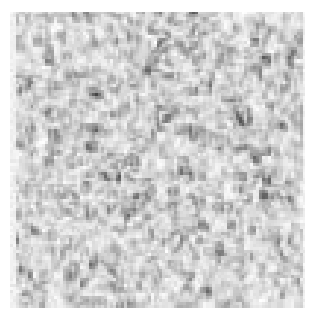

(a)

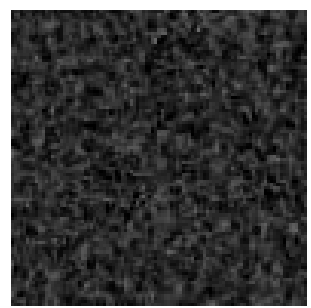

(c)

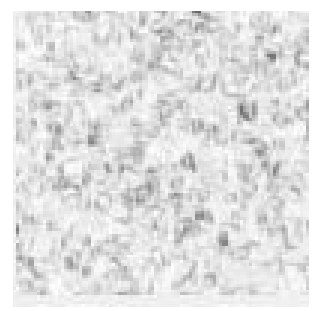

(b)

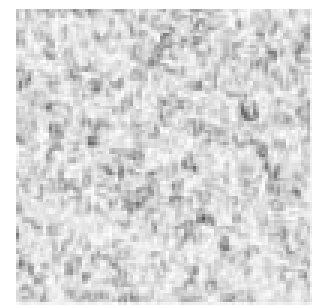

(d)
Figure 2: Similarity maps - (a) 3D vs. 2D; (b) 3D vs. 2D Wiener; (c) 3D vs. 2D Non-local Means; (d) 3D vs. Adaptive Median.

On this map, the white pixels represents identical dots on the compared images, whereas the grey and black dots represent differences. The darker the pixel is, the higher the difference is with, related to luminance, contrast and structure.

\section{Discussions}

The X-ray images are significantly affected by the quantum noise. Thus, in order to improve the image quality, and consequently, the signal to noise ratio, we apply three different digital filters on the images. Table 1 reveals that the filters have behaved differently on the same image, given the different MSSIM index calculated.

The highest similarity was observed on the images under the Wiener filter, which proves how efficient this filter is to reduce the quantum noise. Even though the similarity has increased when the Adaptive Median filter was applied to the $2 \mathrm{D}$ image, proving that its efficiency in reducing the quantum noise is probably lower than when the Wiener filter is used.

These results were shown in similarity maps presented in Figure 2. We noted that the similarity maps between the $3 \mathrm{D}$ image $v s$. Wiener, and $3 \mathrm{D}$ image $v s$. Adaptive Median present lighter gray levels, indicating high similarity.

This increase of similarity between 2D filtered images (Wiener and Adaptive Median) and the 3D image can be explained by the tomosynthesis acquisition geometry. As tomosynthesis reconstructs the imagem from a series of projections, the thickness of the object of study in each projection is smaller, reducing the X-ray scattering, and hence the effect of quantum noise.

In this way, tomosynthesis images are supposed to have more contrast and luminance than de $2 \mathrm{D}$ conventional image due to the lower noise. Therefore, the filters performed a good role, since they increased the similarity, approximating the 2D and 3D images.

The filter Non-local Means resulted in the lowest similarity index calculated, 0.1583 . This filter proved to be inefficient in reducing the quantum noise on the images tested. Figure 2(c) demonstrates this result, since the resultant similarity map is mostly composed of darker values of pixels, revealing a very low similarity.

\section{Conclusion}

On this paper, we were able to quantify the similarity between mammographic image pairs. The use of filters, in some cases, have shown to be efficient to correlate the 2D conventional images and the 3D tomosynthesis image, establishing equivalences regarding luminance, contrast and structure.

For this study, the Wiener filter has apparently been proven a good technique to be used on these types of images, since the noise has been reduced. However, the same not occurred with the Non-local Means filter, as the image with this filter had the lowest similarity to the 3D image.

As a future study, other digital filters will be tested, the number of regions of interest will be expanded and 
images will be collected with different acquisition techniques. We will implement new algorithms in order to establish a comparison with SSIM.

\section{Acknowledgements}

We would like to thank the Radiology Institute of the University of São Paulo for providing us the images and CAPES for the financial support.

\section{References}

[1] Park JM, Franken EA, Garg M, Fajardo LL, Niklason LT. Breast tomosynthesis: Present considerations and future applications 1 . Radiographics. 2007; 27:S231-S240.

[2] Skaane P, Gullien R, Bjorndal H, Eben EB, Ekseth U, Haakenaasen U, Jahr G, Jebsen IN, Krager M. Digital breast tomosynthesis (DBT): initial experience in a clinical setting. Acta Radiologica. 2012; 53(5):524-529.

[3] Baker JA, Joseph YLO. Breast tomosynthesis: state-of-the-art and review of the literature. Academic Radiology. 2011; 18:1298-1310.

[4] Rose SL, Tidwell AL, Bujnoch LJ, et al. Implementation of breast tomosynthesis in a routine screening practice: an observational study. American Journal of Roentgenology. 2013; 200(6):1401-1408.

[5] Ciatto S, Houssami N, Bernardi D, et al. Integration of 3D digital mammography with tomosynthesis for population breast-cancer screening (STORM): a prospective comparison study. 2013; 14(7):583589.

[6] Fornvik D, Zackrisson S, Ljunberg O, et al. Breast Tomosynthesis: Accuracy of tumor measurement compared with digital mammography and ultrasonography. Acta Radiologica. 2010; 3:240247.

[7] Sundaram, DK, Sasikala D, Rani PA. A study on preprocessing a mammogram image using Adaptive Median Filter. International Journal of Innovative Research in Science, Engineering and Technology. 2014; 3(3):10333-10337.

[8] Vieira MA, Bakic PR, Maidment AD, Schiabel, H, Mascarenhas ND. Filtering of poisson noise in digital mammography using local statistics and adaptive wiener filter. In: International Workshop on Digital Mammography, 2012. p. 539-546.

[9] Moradmand H, Setayeshi S, Karimian A, Sirous M. Contrast Enhancement of Mammograms for Rapid Detection of Microcalcification Clusters. Iranian Journal of Medical Physics. 2014; 11: 260-269.

[10] Xu S, Liu X, Jiang S. A Fast Feature Similarity Index for Image Quality Assessment. International Journal of Signal Processing, Image Processing and Pattern Recognition. 2015; 8(11): 179-194.

[11] Dosselmann R, Yang XD. A Formal Assessment of the Structural Similarity Index. University of Regina. Department of Computer Science, 2008.
[12] Okarma K. Combined full-reference image quality metric linearly correlated with subjective assessment. In: Artificial Intelligence and Soft Computing. Springer Berlin Heidelberg, 2010. p. 539-546.

[13] Chen GH, Yang CK, Xie S. Gradient-based structural similarity for image quality assessment. In: IEEE International Conference on Image Processing; 2006 Oct 08-11; Georgia, USA. 2008. p. 3056-3059.

[14] Wang Z, Bovik AC, Sheikh HR, Simoncelli EP. Image quality assessment: From error visibility to structural similarity. IEEE Transactions on Image Processing. 2004; 13(1):600-612.

[15] Vaseghi SV. Advanced digital signal processing and noise reduction. John Wiley \& Sons, 2008.

[16] Buades A, Coll B, Morel JM. A non-local algorithm for image denoising. In: IEEE Computer Society Conference on Computer Vision and Pattern Recognition, 2005. p. 60-65.

[17] Lin HM, Willson AN. Median filters with adaptive length. IEEE Transactions on Circuits and Systems. 1988; 35(6), 675-690.

[18] Casti P, Mencattini A, Salmeri M, Rangayyan RM. Analysis of structural similarity in mammograms for detection of bilateral asymmetry. IEEE Transactions on Medical Imaging. 2015; 34(2): 662-671. 